\title{
The Number Continuity Service: Part I - GSM <-> Satellite Phone
}

\author{
Arnaud Henry-Labordère \\ HALYS, Paris, France and PRISM-CNRS, Versailles, France; e-mail: ahl@halys.fr
}

Received 15 January 2013; Accepted 17 February 2013

\begin{abstract}
Mobile Number Portability is now a widely used service allowing users to keep their number if they change their subscribed operator. It was first deployed in Hong-Kong (1999). "Number Continuity" corresponds to the same service but with the switch to another technology, because the subscribed main terminal does not have coverage. The new terminal may be a PC, a smartphone/WiFi, a "satphone", a GSM phone if the main one is CDMA, with (almost) the same service transparently. Making calls or SMS with its normal CLI shown, receiving calls, SMS, MMS to his normal GSM number (unlike "Skype"). This article explains the GSM <-> satphone number continuity implementation. The Geostationary and Low Earth Orbit characteristics are presented as well the consequences of orbit drifts for which the exact computation is given based on the formal integration of Kepler's area law. The handover implementation and cases for the satellite service are explained. The constellation and services of the main satellite operators are compared. The telecom core network implementation of the service is detailed in the cases of a GSM type core network and an IS-41 (CDMA) core network. Subsequent articles will cover GSM $<->$ CDMA (IS-41) and GSM $<->$ WiFi.
\end{abstract}

Keywords: number continuity, satphone, IS-41, handover, satellite services, IMSI nominal, IMSI auxiliary, multi-IMSI, geostationary, LEO.

Journal of Cyber Security and Mobility, Vol. 1, 349-376.

(C) 2013 River Publishers. All rights reserved. 


\section{User Benefit from the GSM <-> Satellite Number Continuity Implementation}

A customer owns two mobile phones: one GSM mobile and one satellite phone. His contacts know only his GSM number and he will receive few calls to his GSM numbers as no one knows he is using a satellite phone. He is currently in an uncovered GSM area, ship, or open country but his satellite phone is activated after he has turned it on. With the Number Continuity implemented for his satellite phone, he will receive all calls and SMS to his satellite phone even they were sent to his GSM number. The only thing he has done is turning on the satellite phone. No manual call forwarding (it would not forward the SMS) and if he has lost GSM coverage he could not use the call forwarding function. If he is again under GSM coverage, he reactivates it, and again all calls and SMS will be received by the GSM handset.

The Number Continuity Solution allows this transparent service for GSM and Satellite phone customers. It is implemented by satellite operators, or by Roaming Hub operators in cooperation with them and with agreements with the involved GSM operators.

\section{Satellite Coverage and Position Station Keeping of GEO and LEO}

\subsection{Telecom Coverage}

For GEO and LEO the orbit plane is supposed to be the equator (which makes an angle of $23^{\circ} 27^{\prime}$ (the tilt of the earth axis) with the earth ecliptic plane, where all the other planets are mostly (Pluto has an inclined orbit). For a LEO satellite, the orbit is strongly inclined at the equator.

From Newton's gravitation law, we have:

- $\gamma_{g}=K / R^{2}$ for the terrestrial acceleration where $\mathrm{R}$ is the geostationary satellite distance from the earth center. The gravitation field decreases as the square of the distance to the earth center.

- $\gamma_{c}=\omega^{2} \times R$ is the centrifugal acceleration applied to the satellite due to its circular assumed orbit around the center of an earth inertial coordinate system.

The earth (same angular speed exactly as satellite) has a rotation time in one sidereal day $D=23 \mathrm{~h} 56 \mathrm{~m} 4 \mathrm{~s}$ (not exactly the legal 24 hours), which gives 
the common angular speed $\omega$ of the earth and of the geostationary satellite:

$$
\omega=2 \Pi / D=0.000072921173 \mathrm{rad} / \mathrm{sec}
$$

$K$ is such that $\gamma_{g}=9.822 \mathrm{~m} / \mathrm{sec}^{2}$ (the standard gravity) at $R_{0}=6371 \mathrm{~km}$ the official geodetic radius of the "geoïd surface", hence:

$$
K=9.822 \times\left(R_{0}\right)^{2}=3.986714 \times 10^{14} \quad\left(\text { in } \mathrm{m}^{3} / \mathrm{sec}^{2}\right)
$$

Setting the gravitation equal to the centrifugal acceleration $\gamma_{g}=\gamma_{c}$ for a circular orbit, with the above values of $K$ and $\omega$ yields

$$
R=\left(K / \omega^{2}\right)^{1 / 3}=42166 \mathrm{~km} \quad \text { (distance from the earth center) }
$$

which gives an altitude of $R-R_{0}=35795 \mathrm{~km}$ above the standard ground level if the orbit was circular, commonly approximated by $36000 \mathrm{~km}$ and it is independent of the mass of the satellite.

If the orbit was circular and the inclination of the orbit equal to $0^{\circ}$ with the equator, the satellite would look still from a ground telecom station, which is simple for tracking.

The satellite covers the earth within a cone which $1 / 2$ angle is

$$
\arccos \left(R_{0} / R\right)=81^{\circ} \text { latitude }
$$

This is better for telecom coverage of the north zone that most LEO systems with inclined orbits.

\subsection{West-East Drift (Deviation from a Theoretical Circular Orbit)}

When the satellite is launched, the orbit is not exactly circular (this is a delicate and fuel consuming launch phase from an elliptical "transfer orbit") even if we assume that the revolution time is exactly one sidereal day. The satellite has an elliptical orbit (Kepler's ellipse law) with a small excentricity $e(e=0$ for a circle, and $e<0$ for an ellipse). Its equation in polar coordinates is

$$
\rho=P /(1+e \cos \theta)
$$

$P$ is the distance from the focus $F_{1}$ (the centre of the earth) to the ellipse. To test real numerical examples, here are the classical relations between the parameters of an ellipse in polar coordinates and in Cartesian coordinates:

$$
\begin{gathered}
P=a\left(1-e^{2}\right), \text { easy to find, by computing } \rho \text { for } \theta=0 \text { (apogee) and } \\
\theta=\pi \text { (perigee) }
\end{gathered}
$$




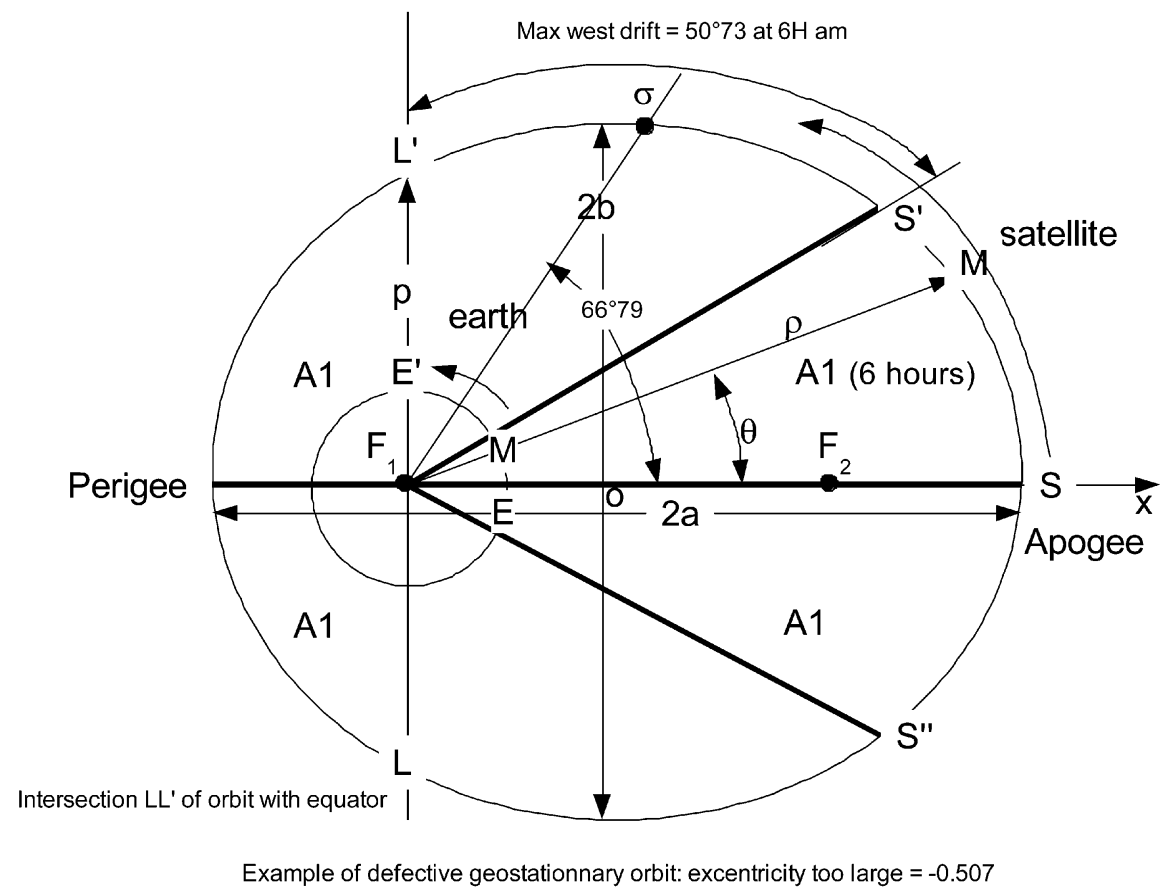

Figure 1 Geostationary orbit with eccentricity $e \neq 0$.

$$
\begin{aligned}
& P=b^{2} / a \\
& c=a e\left(1 / 2 \text { distance between the two focus } F_{1} \text { and } F_{2}\right) \\
& A \text { (the area inside the ellipse) }=\pi a b
\end{aligned}
$$

The perigee (lowest altitude) is $P$ and the apogee (highest) is $A$.

Kepler's law of equal areas says that an equal area of the ellipse is covered in a given time interval. Assume that at $0 \mathrm{~h}$, the satellite is in $S$ vertical of the earth $E$ (the nominal satellite longitude). Six hours later, the satellite is in $S^{\prime}$ (the area $A_{1}=A S^{\prime} F_{1}$ is equal to the area $F_{1} S^{\prime} P$ as $P$ is the satellite position at $12 \mathrm{~h}$ ). At 0,12 and $24 \mathrm{~h}$ again, the satellite is at its nominal longitude. However, in the $A S^{\prime}$ part of the day, the satellite is revolving slower that the earth and appears to drift westward (the earth turns west to east). When the satellite is in $S^{\prime}$, the drift difference is the angle $E^{\prime} F_{1} S^{\prime}$. In the $S^{\prime} P$ part, it is faster and appears to drift eastward. An earth observer sees the satellite drifting westward from $0 \mathrm{~h}$ to $T$ and coming back to its nominal longitude from $T$ to $12 \mathrm{~h}$, drifting eastward from $12 \mathrm{~h}$ to $(24 \mathrm{~h}-T)$ and coming back to its nominal longitude from $24 \mathrm{~h}-T$ to $24 \mathrm{~h}$. 
If the orbit is far from being circular, the $E-W$ telecommunication coverage may be defective during the two daily periods of maximum drift.

\subsubsection{Angular Position $\theta(t)$ of the Satellite}

The total area $A$ of the ellipse can be computed simply as above knowing $P$ and $e$. The differential of $A$ for a small angle $\theta$, is

$$
\left.d A=\frac{1}{2} P^{2} d \theta \quad \text { (area of a small triangle with height } \rho \text { and basis } \rho d \theta\right)
$$

To get $\theta(t)$ we use Kepler's equal area law.

Assuming to simplify that the angular position of the satellite is $S=0$ at $0 \mathrm{~h}$ we have

$S \frac{1}{2}\left(P^{2}(1-e \cos \theta)^{2}\right) d \theta=(A / D) t$ the area of the ellipse from 0 to $t$ time $) 0, t$

Kepler's law: Area between time 0 and $T=\int_{0}^{T} \frac{P^{2}}{(1+e \cos \theta)^{2}} d \theta=k T$ (2)

Taking $T=D$ (full revolution in one day $23 \mathrm{~h} 56 \mathrm{~m} 4 \mathrm{~s}$ ), the total ellipse area being $A$,

$$
A=k D \rightarrow k=\frac{A}{D}
$$

This is the integral equation relating to time $T$ and the angular position $\theta(T)$.

It is complicated to integrate by hand but this is a "definite integral" (using elementary functions), the online formal integration tool [3] gives the result using real valued functions only for $|e|>1$ so the trick is to set the eccentricity $e^{\prime}=1 / e$ in [2] and do instead a formal integration of

$$
g(\theta)=\int \frac{1}{\left(1+\left(\frac{1}{e}\right) \cos \theta\right)^{2}} d \theta
$$

which gives an expression of real valued functions $\left(e^{2}-1\right.$ is $\left.>0\right)$ :

$$
e^{2}\left(\frac{2 e \tan ^{-1}\left(\frac{(e-1) \tan \left(\frac{\theta}{2}\right)}{\sqrt{e^{2}-1}}\right)}{\left(e^{2}-1\right)^{3 / 2}}-\frac{\sin (\theta)}{\left(e^{2}-1\right) \cos (\theta)+e}\right)
$$


Using Kepler's area law [2], the value of $k$ in [3] and the expression of $g(\theta)$ [5] yields

$$
g(\theta(T))=\frac{A}{D P^{2}} T
$$

This gives

$$
\theta(T)=g^{-1}\left(\frac{A}{D P^{2}} T\right)
$$

(but $g^{-1}$ can only be computed numerically).

\subsubsection{Program to Compute the Time $T$ to Have the Angle ${ }^{1}$}

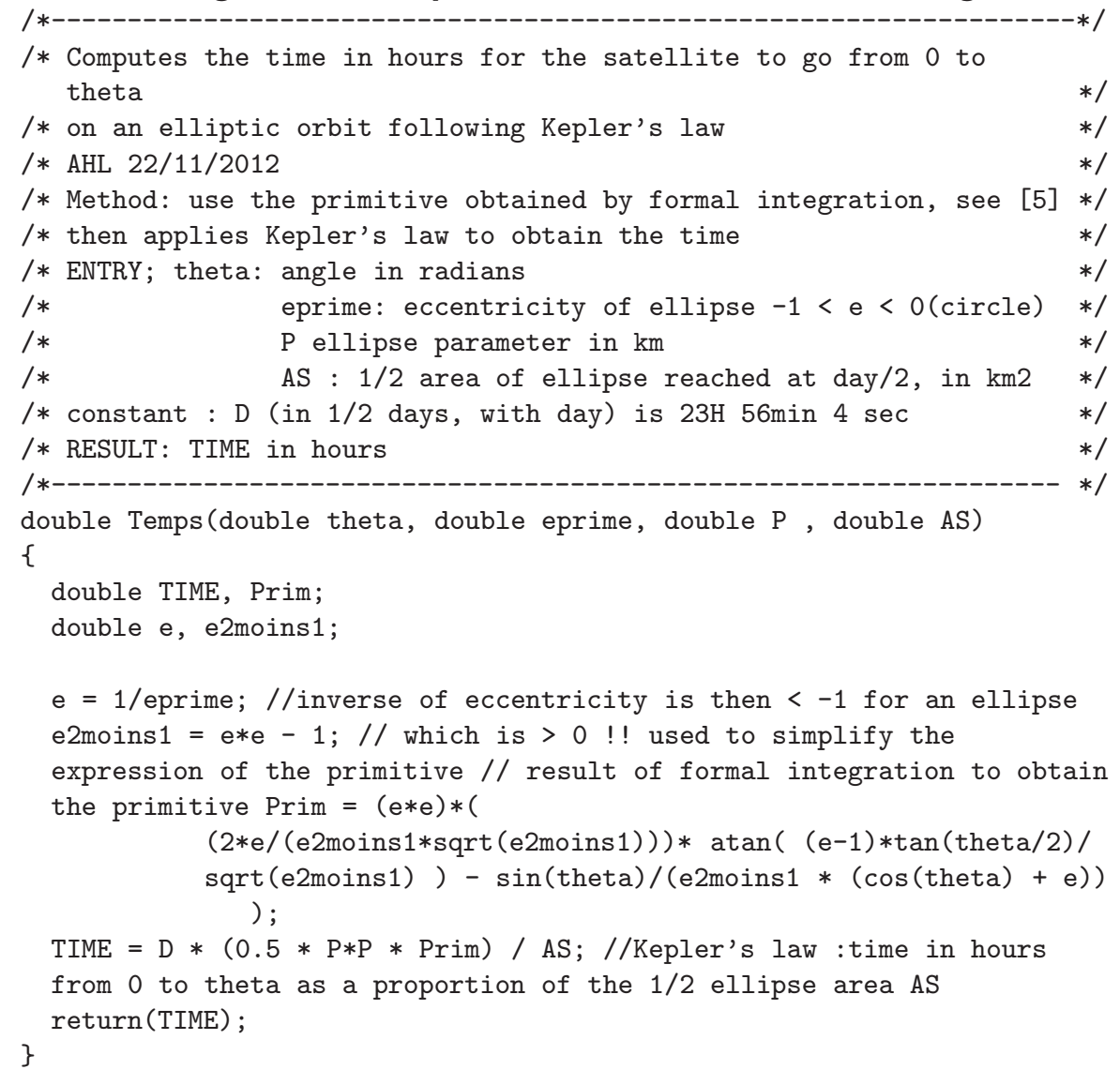

1 Thanks to Pascal Adjamagbo and Jean-Yves Charbonnel (Institut de Mathématiques de Jussieu (IMJ), November 2012. 


\subsubsection{Computation of Angle of Radial Speed Synchronism}

The radial speed of the satellite and of the earth are the same for an angular position $\theta_{s}$ such as

$$
\frac{P^{2}}{\left(1+e \cos \theta_{s}\right)^{2}}=\frac{A}{\pi}
$$

that is,

$$
\theta_{s}=\arccos \left(\frac{P \sqrt{\frac{\pi}{A}}-1}{e}\right)
$$

which we represent as $\sigma$ in Figure 1.

\subsubsection{Computation of the Maximum Westward and Eastward Daily Drift}

The drift is the difference in longitude between the normal longitude of the geostationary satellite and the real longitude.

$\theta_{s}=66^{\circ} 79^{\prime}$ with the above numerical values. At this angular position, the west drift is maximum because the satellite becomes faster eastward than the earth. The numerical integration gives $t_{s}=8 \mathrm{~h} 27 \mathrm{~m}$. We can compute the earth angular position and the drift.

Normally, the tolerance given by the space organisations for geostationary station keeping is about $1^{\circ}$ which means that if the launch does not succeed in setting an almost circular orbit, the satellite is not usable. Some telecom operators

\subsubsection{Long-Term West or East Drift}

The drift is due to the earth potential not being symmetrical (the equator is slightly elliptical), and has a tendency to "pull" the latitude toward two stable

\begin{tabular}{|c|c|c|c|c|}
\hline Excentricity & $\begin{array}{l}S^{\prime}=\text { maximum } \\
\text { westward drift } \\
\text { at } 6 \mathrm{~h} \mathrm{am}\end{array}$ & $\begin{array}{l}\text { Duration of } \\
\text { north drift } \\
=g(\pi / 2-0)\end{array}$ & $\begin{array}{l}\text { Duration of } \\
\text { south drift } \\
=g(\pi-\pi / 2)\end{array}$ & $\begin{array}{l}\text { Angular } \\
\text { position of } \\
\text { synchronism }\end{array}$ \\
\hline-0.507 & $60^{\circ} 08$ & $9 \mathrm{~h} 42 \mathrm{~m}$ & $2 \mathrm{~h} 18 \mathrm{~m}$ & $66^{\circ} 79$ \\
\hline$\ldots$ & .. & .. & $\ldots$ & .. \\
\hline-0.017 & $1^{\circ} 95$ & $6 \mathrm{~h} 8 \mathrm{~m}$ & $5 \mathrm{~h} 52 \mathrm{~m}$ & $89^{\circ} 27$ \\
\hline-0.012 & $1^{\circ} 38$ & $6 \mathrm{~h} 5 \mathrm{~m}$ & $5 \mathrm{~h} 55 \mathrm{~m}$ & $89^{\circ} 48$ \\
\hline-0.007 & $0^{\circ} 80$ & $6 \mathrm{~h} 3 \mathrm{~m}$ & $5 \mathrm{~h} 57 \mathrm{~m}$ & $89^{\circ} 69$ \\
\hline-0.002 & $0^{\circ} 23$ & $6 \mathrm{~h} 1 \mathrm{~m}$ & $5 \mathrm{~h} 59 \mathrm{~m}$ & $89^{\circ} 91$ \\
\hline 0 (circle) & $0^{\circ}$ & $6 \mathrm{~h}$ & $6 \mathrm{~h}$ & All \\
\hline
\end{tabular}

Table 1 West-east and north-east drift depending on the orbit's eccentricity. 
"tesseral points" (from the harmonic function theory of Simon Laplace) at $75^{\circ} \mathrm{E}$ and at $104^{\circ} \mathrm{W}$, with two unstable "tesseral points" at $165^{\circ} \mathrm{E}$, and at $14^{\circ} \mathrm{W}$. Regular fuel consuming corrections must be done from the longitude station keeping.

\subsection{North-South Drift (Orbit Not in the Equatorial Terrestrial Plan)}

The launch may be such that the satellite orbit makes a small angle $\gamma$ with the equator plane. In the figure the intersection of the orbit with the equator is $L L^{\prime}$ (we assumed for simplicity that the apogee of the orbit was at the nominal longitude). The maximum north latitude deviation is ?at $0 \mathrm{H}$ and south at $12 \mathrm{~h}$. It remains north from $A$ (apogee) to $L^{\prime}$ and south from $L^{\prime}$ to $P$ (perigee). The time during which there is a north-south drift is also computed numerically in Table 1, for the north drift it is the time when $\theta=\pi / 2$ starting from the apogee $P$ at $0 \mathrm{~h}$. For $e=-0.507$ the satellite has a $0^{\circ}$ latitude at $9 \mathrm{~h} 42 \mathrm{~m}$ and $14 \mathrm{~h} 18 \mathrm{~m}$.

\subsubsection{North-South Deviation during the Day}

The north-south drift is the "declination" $\delta$ of the satellite (corresponding to $V M$ ) for a given angle $\theta$. The spherical triangle $L^{\prime} V M$ (with a rectangular angle $V$ such as $\sin V=1$ ) has the well-known spherical trigonometric relation:

$$
\begin{gathered}
\sin \gamma / \sin \delta=\sin V / \sin \left(\frac{\pi}{2}-\theta\right) \\
\sin \delta=\sin \gamma \cos \theta
\end{gathered}
$$

("declination" $\delta$ of satellite as a function of its angular position $\theta$ ).

\subsubsection{Long-Term North-South Drift}

The combined effect of the sun gravitation mainly, of the moon and (the earth potential not being spherical being the least important) tends to "pull" the orbit plane to align with the ecliptic plane earth-moon-sun at a speed of about $1 \%$ year. The fuel budget to maintain the latitude is much more than the longitude station keeping (about $50 \mathrm{~m} / \mathrm{sec}$ against $2 \mathrm{~m} / \mathrm{sec}$ ) speed impulses. 


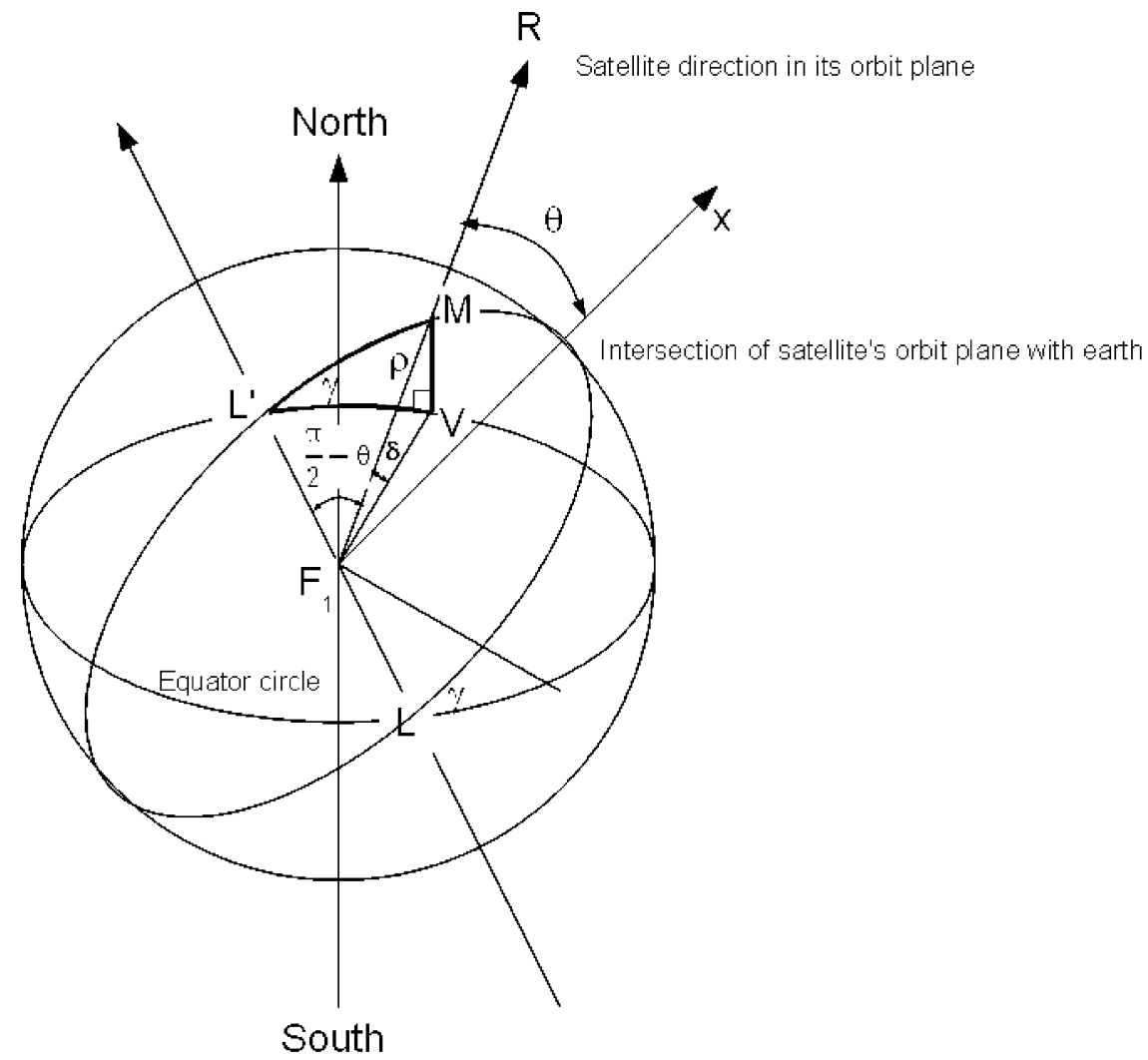

Figure 2 Spherical triangles used in the orbit computation.

\subsection{Daily Ground Trace of the "Geostationary" Satellite (Ephemeris)}

Not counting the long-term drifts, the ground trace is a combination of the $24 \mathrm{~h}$ periods longitude and latitude drifts. In the simplified example (we assume the satellite being exactly at his nominal longitude at the apogee (and perigee). We have, using [7],

- Longitude drift $=(2 \pi T / D)-g^{-1}\left(\frac{A}{D P^{2}}\right) T$ (difference at time $T$, between the earth angular position and satellite's which is $\theta(T)$ )

- Latitude drift $\left.=\delta(T)=\arcsin \left(\sin \gamma \cos g^{-1}(T)\right)\right)$ 


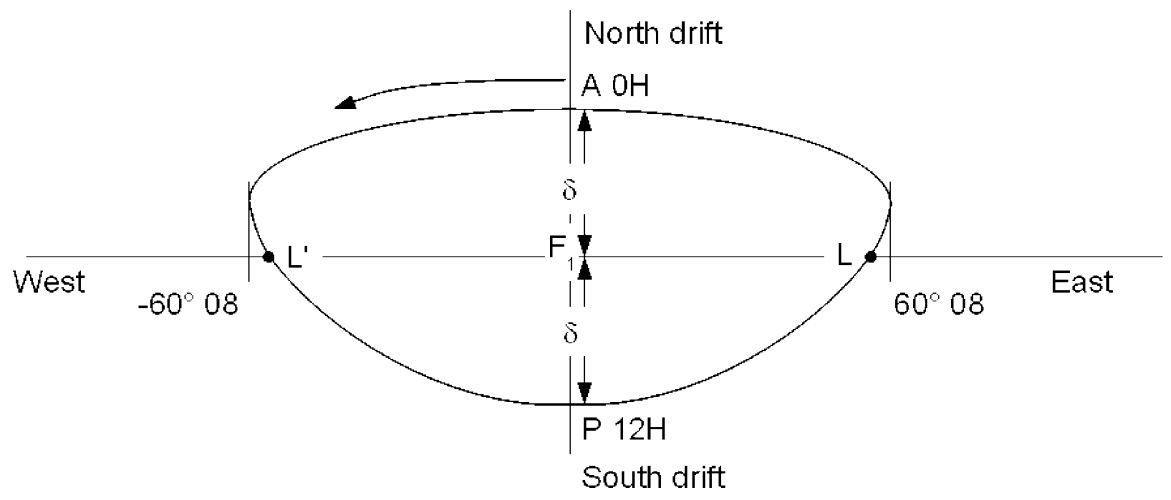

Figure 3 Ground trace of geostationary satellite.

that is, using also [7]

$$
\text { Latitude drift }=\delta(T)=\arcsin \left(\sin \gamma \cos g^{-1}\left(\frac{A}{D P^{2}} T\right)\right)
$$

If one wanted to use a satellite to make a traditional computation of its own position with a sextant and accurate watch, the curve gives the ephemeris coordinates $\alpha$ and $\delta$ of the satellite.

We gave an elementary but exact 2 body model (earth and satellite) of the orbit computation. In [5, vol. I], there is the more accurate model using the " $n+1$ body" perturbation theory of Lagrange which is used for long term planet orbit computations and operational satellite ephemeris. When the orbit is close to the simple 2 body model, there is a system of $3 n$ second order non linear differential equations, which is numerically computed. (FrançoisXavier Lagrange who invented the method (1736-1813) must have had a hard time properly solving [he was the first] the 3 body problem earth-sun-moon.)

\section{Handover in Satellite Calls}

Handover is the procedure which allows a call to proceed without interruption when the satellite handset coverage conditions change due to the serving satellite quick change of position, movement of the handset, or atmospheric conditions. 


\subsection{Geostationary Systems (Inmarsat (Isatphone), Thuraya)}

Each of the satellites may be considered equivalent to a BSC (RNC). Each of the MSC-VLR corresponds to a single ground station with a single BSC. There is a handover when the user moves further East or West than the coverage of the current satellite. The procedure is exactly the standard "inter-MSC handover" [2]. The MSC of the new ground station A sends a MAP_PERFORM_HANDOVER to the previous MSC B giving the next cell where the call must be transferred. It is exactly like GSM and described below for the LEO Inter-gateway handover case.

\subsubsection{LEO Systems (Iridium Example)}

What is called a "beam" of these systems has a given frequency and covers a "spot" (earth footprint) for the subscribers. Each antenna (e.g. Iridium case) has 16 "beams", so a satellite has a total of 48 . Each spot, which may overlap, may be covered by several beams (frequencies). The satellites which have quickly varying positions are connected for control and for relay of communications to a network of XX ground stations, a "satellite gateway", which also are connected to the fixed lines PSTN (calls out and in) and together (inter-MSC handover) by SS7 signalling links through the satellite constellation.

We will explain the LEO handover procedures based on the readers' assumed knowledge of the GSM handover procedures, with a little refresh. For this comparison: "satellite gateway" = MSC-VLR, each satellite = BSC, each "beam" = cell covered by a BTS.

3.1.1.1 Intra-Beam Handover, Equivalent to GSM "Intra-Number Handover". As the quality of the signal degrades, the handset may monitor that another frequency is better ("intra-beam handover" at the initiative of the handset) or the satellite wants he handset to use an other frequency because it may interfere with another satellite's beam. In this case the satellite asks the handset to change the frequency.

3.1.1.2 Inter-Beam Handover [1], Equivalent to GSM "Inter-Number Handover" [2]. The handset decides to switch to another beam of the same satellite because the signal quality is better. In GSM this is equivalent to a handset going to another cell. It sends a request to the BSC and the BSC manages the change including to a cell it has selected. 


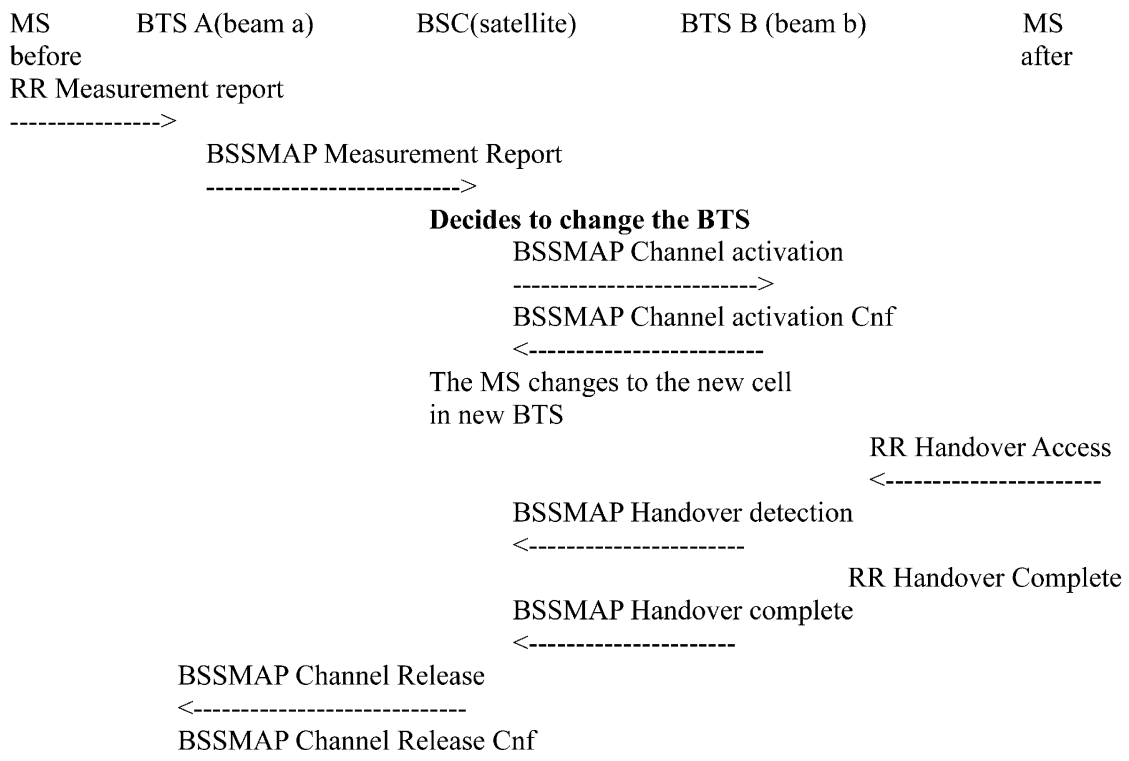

3.1.1.3 Inter-Satellite Handover (Term in [1]), Equivalent to GSM "Intra-MSC Handover" (term in [2]). The serving satellite is moving and the spot where the handset is making a call may not be covered well any more. The serving gateway A which is used for the call asks a new satellite under its coverage to pursue the call with the handset. The connection of the handset with the previous satellite is released. The gateway acts as a MSC-VLR in "the inter-MSC handover" case (same MSC, new BSC) which is recalled below (it is not standard BSSAP which is used for satellites of course). 
The Number Continuity Service. Part I 361

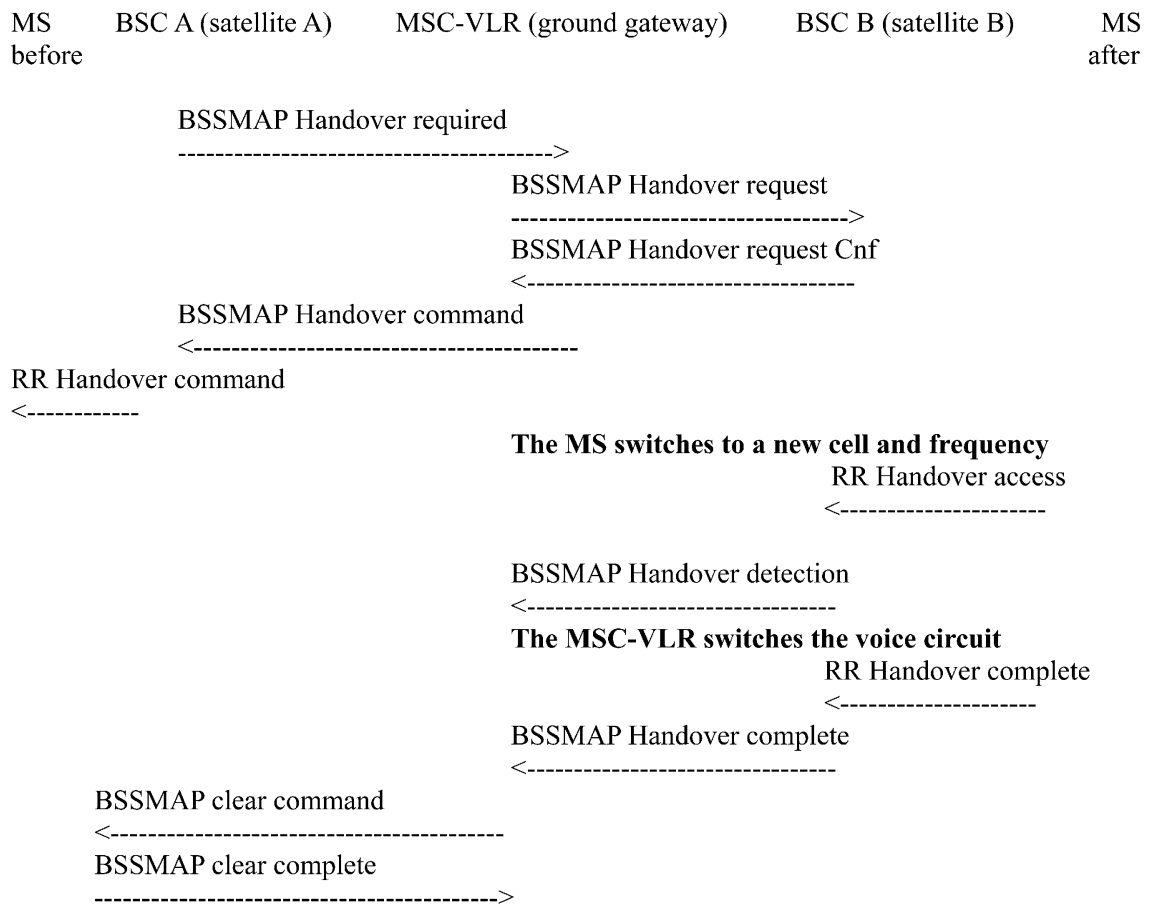

The radio equivalent of the well known SCCP is called the protocol RR. As you can see, there is no use of the MAP protocol as only one MSC-VLR is involved and MAP is used only for Handover between MSC-VLR as in the inter-MSC handover below.

3.1.1.4 Inter-Gateway Handover, Equivalent to GSM "Inter-MSC Handover [2]. Due to the movement of the handset, if it is not well covered any more by a satellite covered by the current ground gateway ground gateway where the call was established ("anchor gateway"). This case exists for satellite but his not described in [1]. When a handset finds a better coverage with an other satellite it will start the same as above, giving the identity of the new satellite (new LAC, Cell Id in GSM) to his current service gateway A.

In GSM it will be as given below: 


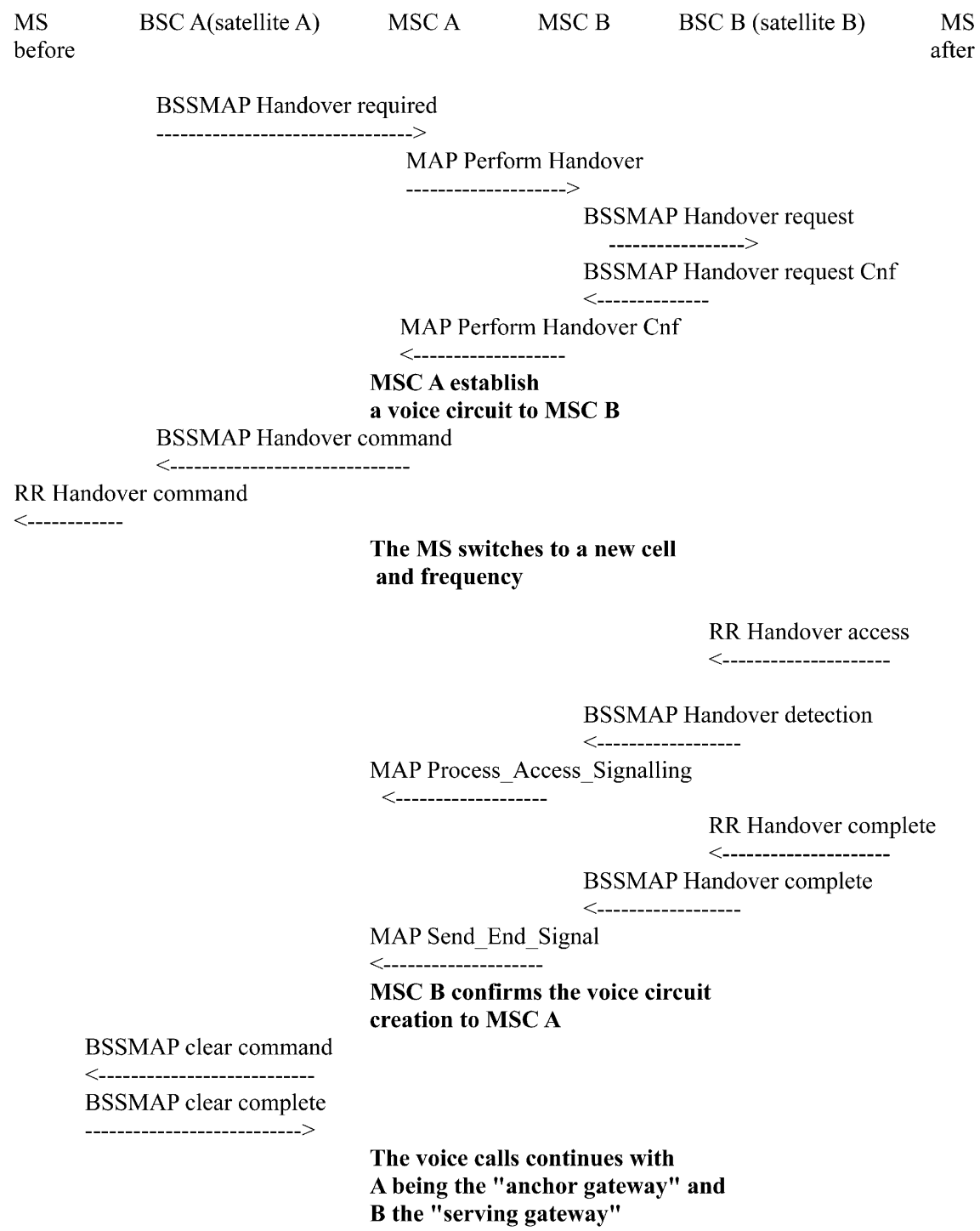

The call is "tromboned" using the voice pass through the satellites as illustrated in Figure 4. 


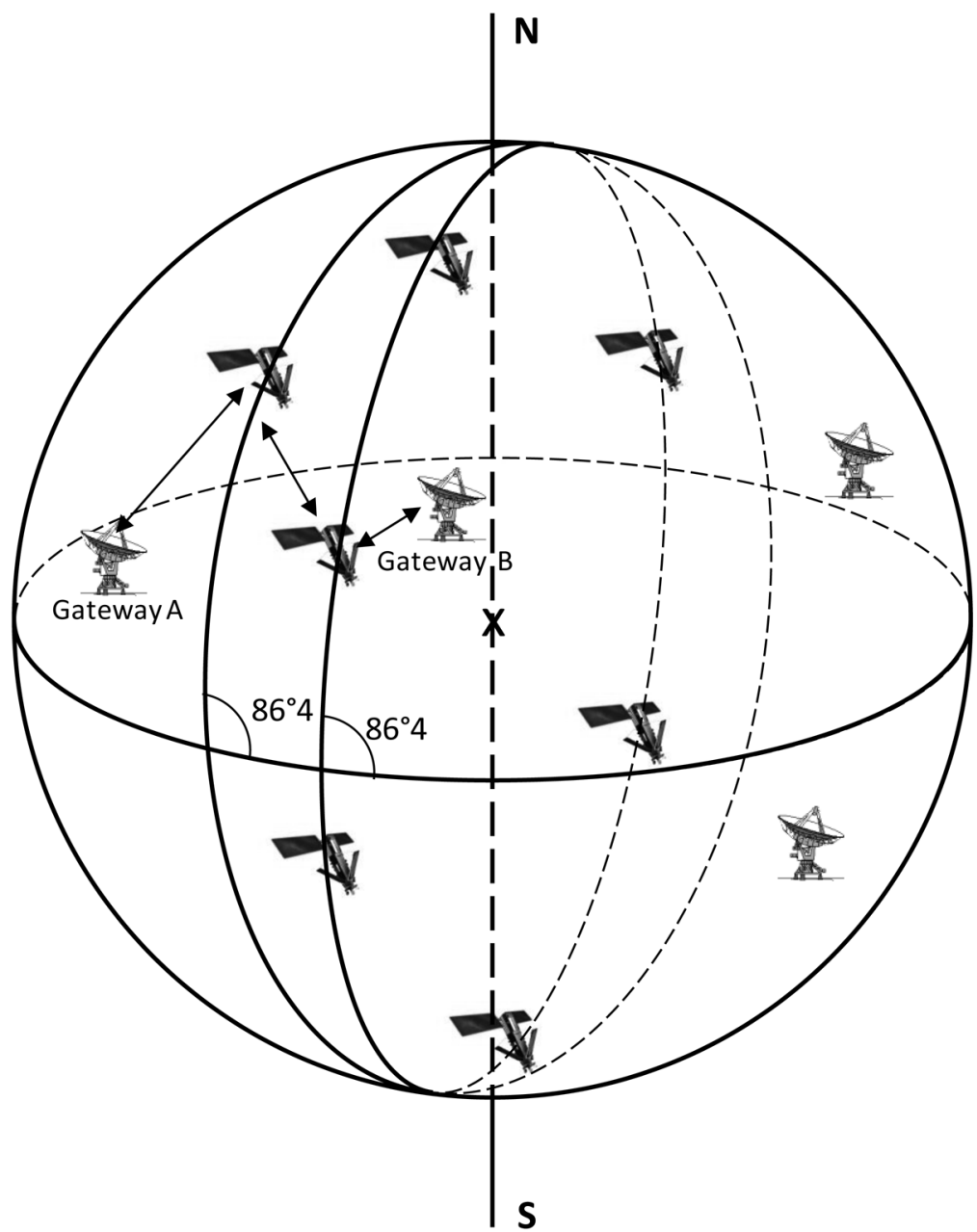

Figure 4 Example of a satellite constellation.

\section{Satellite Operators Concerned by the Number Continuity Service}

They provide special handsets with a direct radio connection to satellites. In most cases they also operate a satellite network, either geostationary (GEO) with an equatorial orbit or a constellation of Low Earth Orbit satellites with inclined orbits. Below the explain the basis of satellite dynamics and also the principle of telecom space networks with a constellation of LEO space- 
crafts when the handover is required to maintain the connections (Iridium, Globalstar).

\subsection{Some Satellite Handsets and Core Networks}

They are easily recognizable by their voluminous foldable antenna. To be practical, it is easy to see that the handsets below have big antennas, they do not look as compact GSM.

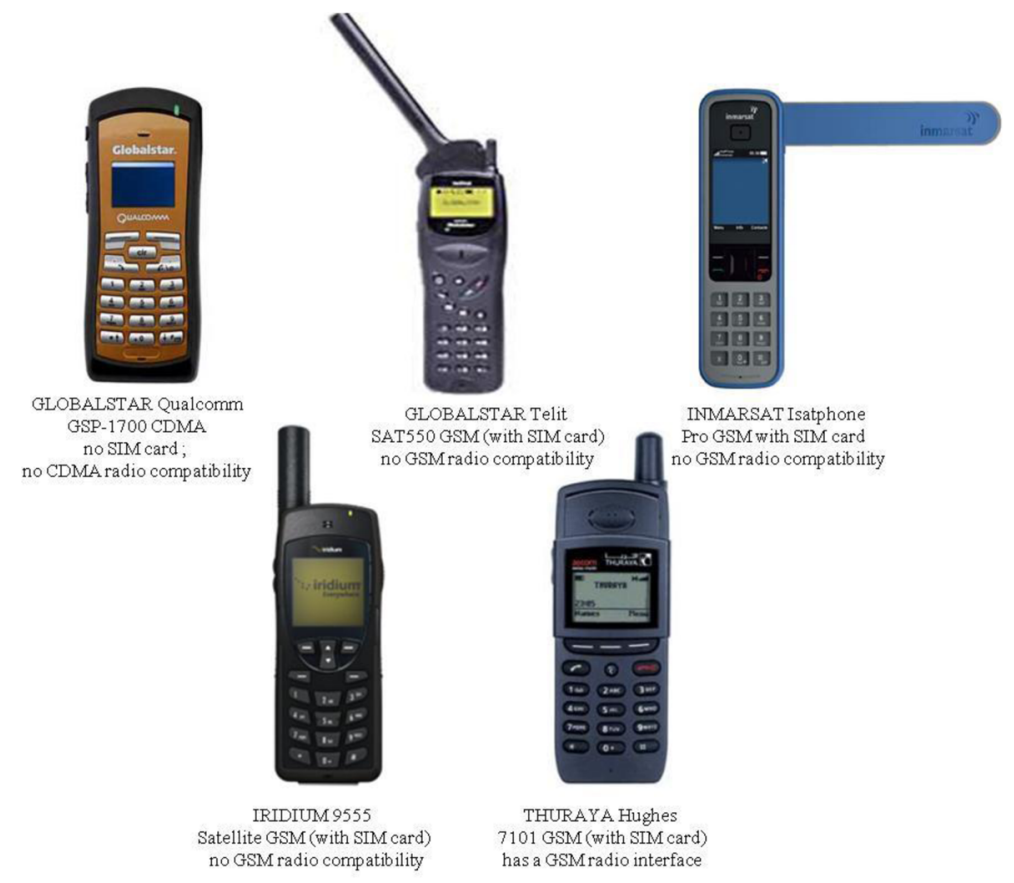

The SIM card terminals accept GSM cards from networks if there is roaming agreement. The user is invoiced by his own network. Only the Thuraya handset is usable on fixed GSM network,

- with GSM or CDMA compatibility (roaming possibility with terrestrial networks):

- Globalstar (LEO constellation) (GSM with SIM card or CDMA, only the CDMA handset is commercialized any more) (commercialization and national gateways, e.g. TESAM (GSM) France closed in 2001, see list in above tables).

- Thuraya (GSM). 
The Number Continuity Service. Part I 365

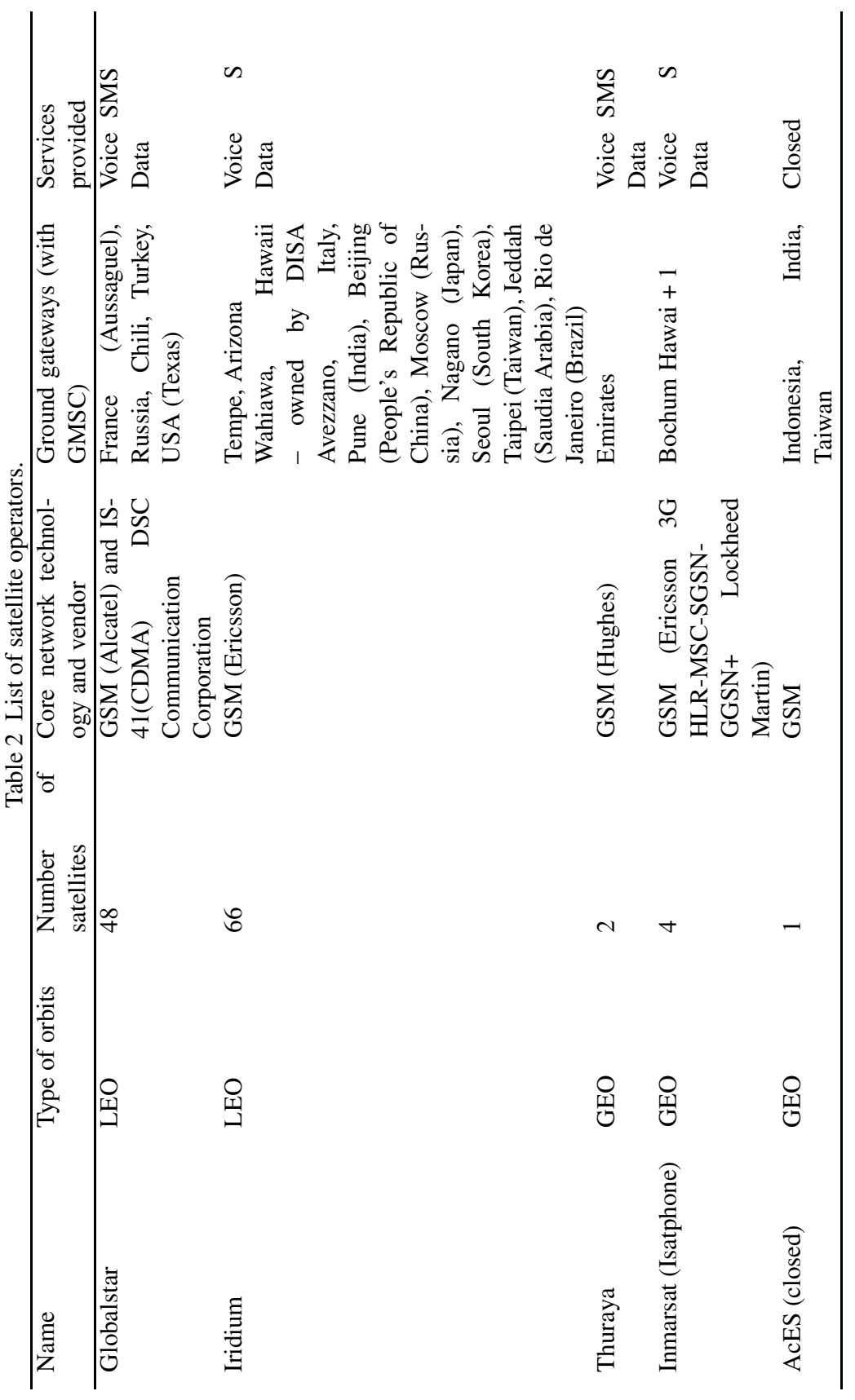


- Innmarsat (C and M terminals), and Isatphone handsets with SIM card.

- Thuraya (GSM) with SIM cards.

- AcES (GSM) with SIM cards (closed in 2004), service taken by Inmarsat with new satellites and ground infrastructure under the Isatphone brand.

The GSM handsets have a SIM card and are dual mode GSM and satellite radio transmission. Technically (Thuraya does it), they can visit a terrestrial GSM network if they have the roaming agreement.

- without GSM or CDMA compatibility (cannot use terrestrial networks)

- Iridium (constellation)

\subsection{Operators Not Concerned by the Need for Number Continuity: Air, Sea (Maritime) and GSM "Bubble Service" and Satellite Operators without Voice Services}

Contrary, ONAIR, AeroMobile, Meagafon which provide the GSM service (with "femtocells" in the aircraft or ships are not concerned by "number continuity" unless they use WiFi. The ships are equipped with classical BTS and leaking lines are used as antennas. There is a satellite radio link (Inmarsat mostly) with the ground segment. This allows using the standard GSM handsets. Astrium is a "GSM bubble service" provider; they install ground BTS with satellite links (their Astrium or Inmarsat ) to their core GSM network (HLR, MSC and IN).

On the GSMa site (2012), in the categories Air there is: ONAIR, Aeromobile and MegaFon. In the Sea category: 7 operators, Maritime Partners, ATT (Wireless Maritime), Siminn (On Wave), ONAIR(OnMaritime), Seanet, Smart Coms(Blue Ocean), Telecom Italia. Also even if Intelsat is a major satellite operator, they are not concerned (no handset) neither the access providers such as Satcom.

However the Air, Sea and "bubble" operators are concerned by the alternative use of WiFi by smartphones to provide the same service as GSM.

Also even if Intelsat is a major satellite operator, they are not concerned (no handset) neither the access providers such as Satcom. 
Table 3 Satellite vendors for the satellite telephone service.

\begin{tabular}{|c|c|c|}
\hline Networks & Satellites & Designer/vendor of mobile \\
\hline $\begin{array}{l}\text { Globalstar (roaming entre } \\
\text { les Gateways Globalstar): } \\
\text { on peut avoir un numéro } \\
+336400 x \text { et s'en servir en } \\
\text { Australie }\end{array}$ & $\begin{array}{l}52 \text { Loral satellites (origin- } \\
\text { ally) } 32 \text { Thalès Alenia (new } \\
\text { generation), inclined } 52^{\circ} \text { in } \\
8 \text { orbit planes }\end{array}$ & $\begin{array}{l}\text { Qualcomm (based on a } \\
\text { CDMA handset, no Global- } \\
\text { star SIM card, the MSISDN } \\
\text { Globalstar is wriiten in the } \\
\text { handset } \\
\text { Telit (GSM) }\end{array}$ \\
\hline Iridium & $\begin{array}{l}\text { LEO (near polar, inclined } \\
86^{\circ} 4 \text { ), only system cover- } \\
\text { ing polar regions; } 66 \text { satel- } \\
\text { lites in } 11 \text { orbit planes } \\
\text { Thalès Alenia (new genera- } \\
\text { tion) }\end{array}$ & \\
\hline Thuraya & Boeing (2 satellites) & ASCOM and Hughes \\
\hline Inmarsat & $\begin{array}{l}3 \text { Astrium EADS ( } 4 \text { I- } 4 \text { and } \\
7 \text { I- } 2 \text { or I-3) }\end{array}$ & Elcoteq (Estonia) \\
\hline $\begin{array}{l}\text { AcES (closed 2011) had } \\
\text { roaming with: } \\
\text { Hong Kong CSL } \\
\text { Hutchison Telecom (HK) } \\
\text { Bharti Hexacom Ltd (AIR- } \\
\text { TEL) } \\
\text { Excelcomindo (Indonesia) } \\
\text { PT Indonesian (INDOSAT) } \\
\text { PT Telekomunikasi Selular } \\
\text { (TELKOMSEL) } \\
\text { Safaricom (Kenya) } \\
\text { DiGi Telecommunications } \\
\text { (Philippines) } \\
\text { SingTel Mobile Singapore } \\
\text { Dialog Axiata (Sri Lanka) } \\
\text { Swisscom (Switzerland) }\end{array}$ & $\begin{array}{l}\text { "Garuda" was the satellite } \\
\text { name }\end{array}$ & Ericsson \\
\hline
\end{tabular}

\subsubsection{Satellite Data Services (No Voice)}

\begin{tabular}{|c|c|c|}
\hline Operator & Type of satellites & Type of service \\
\hline Intelsat & 66 GEO & \\
\hline Orbcomm & $\begin{array}{l}29 \text { LEO }(775 \mathrm{~km}) \text {. Small satel- } \\
\text { lites }(50-120 \mathrm{~kg}) \text { launched mid } \\
1990 \mathrm{~s}\end{array}$ & $\begin{array}{l}\text { Small amount of data (mes- } \\
\text { sages) and Automatic Identific- } \\
\text { ation System (simple devices). } \\
\text { All boats in the Vende Globe } \\
\text { race are equipped with an IAS } \\
\text { which helps to avoid collisions }\end{array}$ \\
\hline
\end{tabular}




\subsection{Coverage and Details of the Various Satellite Operators}

\subsubsection{Globalstar}

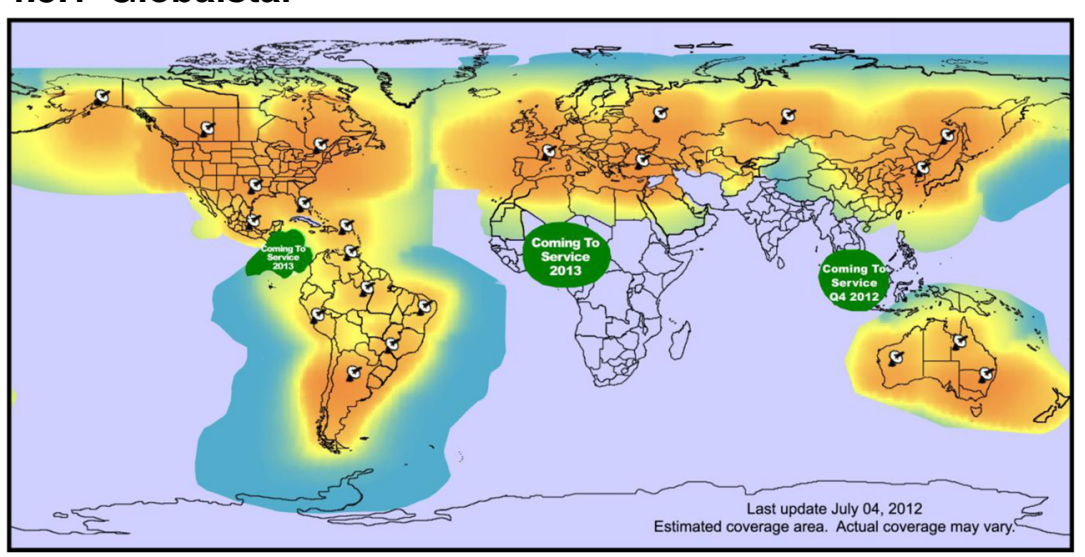

http://www.globalsatellitecommunications.com/globalstar/coverage_map.html

\subsubsection{Iridium}

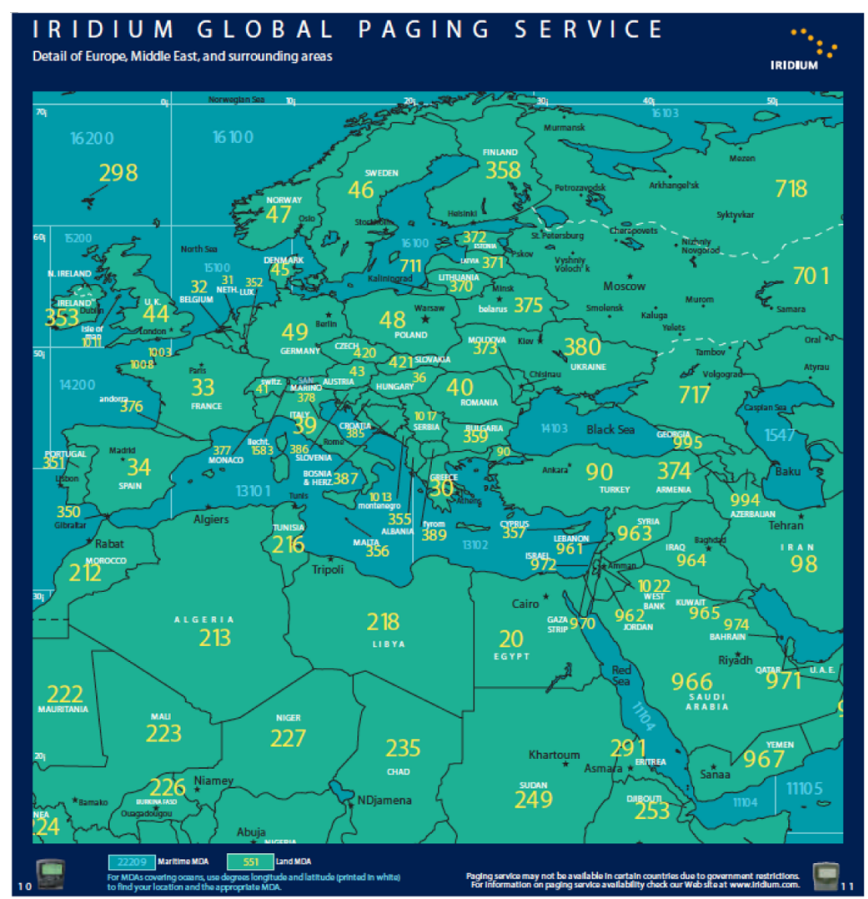

http://www.iridium.com/support/library/CoverageMaps.aspx 
- Iridium SSC, Iridium communications service was launched on November 1, 1998. Motorola provided the technology and major financial backing.

- Chapter 11 bankruptcy nine months later, on August 13, 1999.

- Service was restarted in 2001 by the newly founded Iridium Satellite LLC, which was owned by a group of private investors.

The Iridium constellation is the largest in the world, with 66 low earth orbiting (LEO) satellites operating as a fully meshed network. Iridium flexible billing and flat rates for calls from anywhere to anywhere on earth.

\section{GSM-Like Offer}

Removable Subscriber Identity Modules (SIMs) are used in Iridium phones, much like those used for GSM. Prepaid SIM cards are usually green while post-paid cards are red.

Iridium operates at only 2.2 to $3.8 \mathrm{kbit} / \mathrm{s}$, which requires very aggressive voice compression and decompression algorithms. Latency for data connections is around $1800 \mathrm{~ms}$ round-trip, using small packets.

There is a Web/e-mail to an SMS gateway which enables messages to be sent from the Internet or an e-mail account to Iridium handsets for free. There is also a voice mail service.

\section{Tracking Transceiver Units}

Without an extra GNSS receiver tracking is difficult, but not impossible, as the position of a mobile unit can be determined using a Doppler shift calculation from the satellite. These readings however can be inaccurate with errors in the tens of kilometres. Even without using Doppler shifts, a rough indication of a unit's position can be found by checking the location of the spot-beam being used and the mobile unit's timing advance.

The position readings can be extracted from some transceiver units and the 9505A handset using the -MSGEO AT command.

\subsubsection{Thuraya}

Thuraya, is an international mobile satellite services provider, based in Abu Dhabi and Dubai, the United Arab Emirates, and covering mainly the Middle East, Africa, Western Europe, Asia and the Australia.

The system allows telecommunications in voice, data and SMS. The services also provide the GmPRS for direct access to the Internet. 
Several models are available; they allow the connection by satellite as well as the GSM networks.

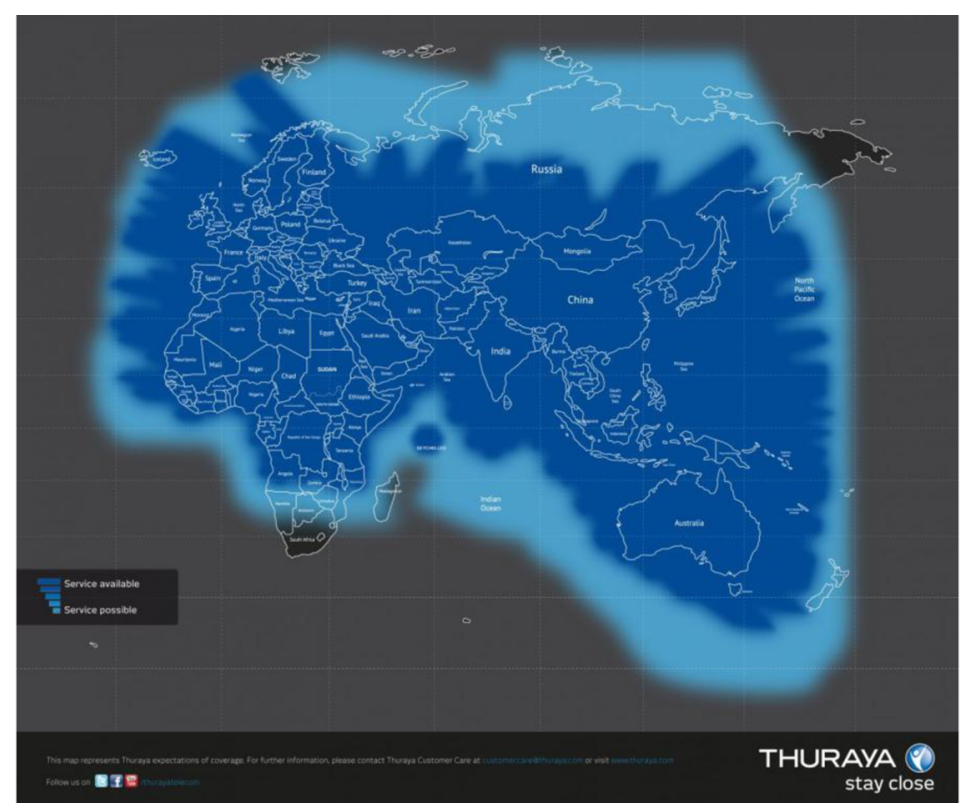

http://www.thuraya.com/coverage-map

\subsubsection{Inmarsat}

Inmarsat Coverage Foot Print

The MAP gives the number of the "spots" which can be used to get a very rough estimate of the handset position with the timing advance.

\section{Systematic Legal Interception}

USA, Russia, India et China enforce that the aircraft using GSM/ Inmarsat, when they fly over their territory have all the voice and data communications "tromboned" through their monitoring system before coming back to the ground station concerned. This is automatic as the satellite links transmit permanently the coordinates of the aircraft and the ground station, which has a numerical map, automatically establishes and suppresses the "tromboning".

The satellites are digital transponders that receive digital signals, reform the pulses, and then retransmit them to ground stations. 
Ground stations maintain usage and billing data and function as gateways to the public switched telephone network and the Internet.

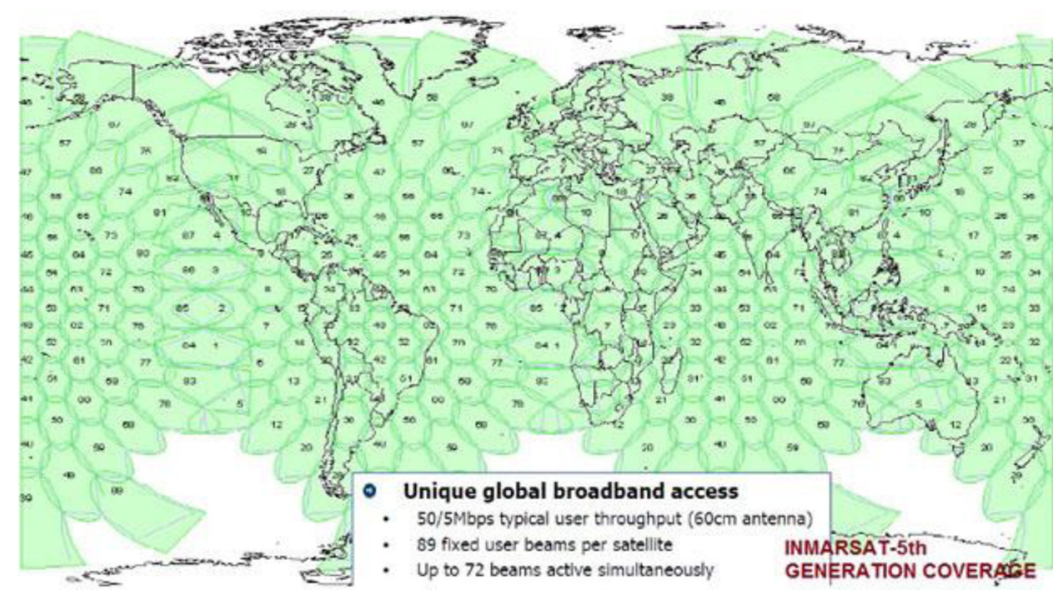

http://www.inmarsat.com/cs/groups/inmarsat/documents/document/016329.pdf http://www.groundcontrol.com/Global_Xpress_Coverage_Map.htm

Teleports and Satellites

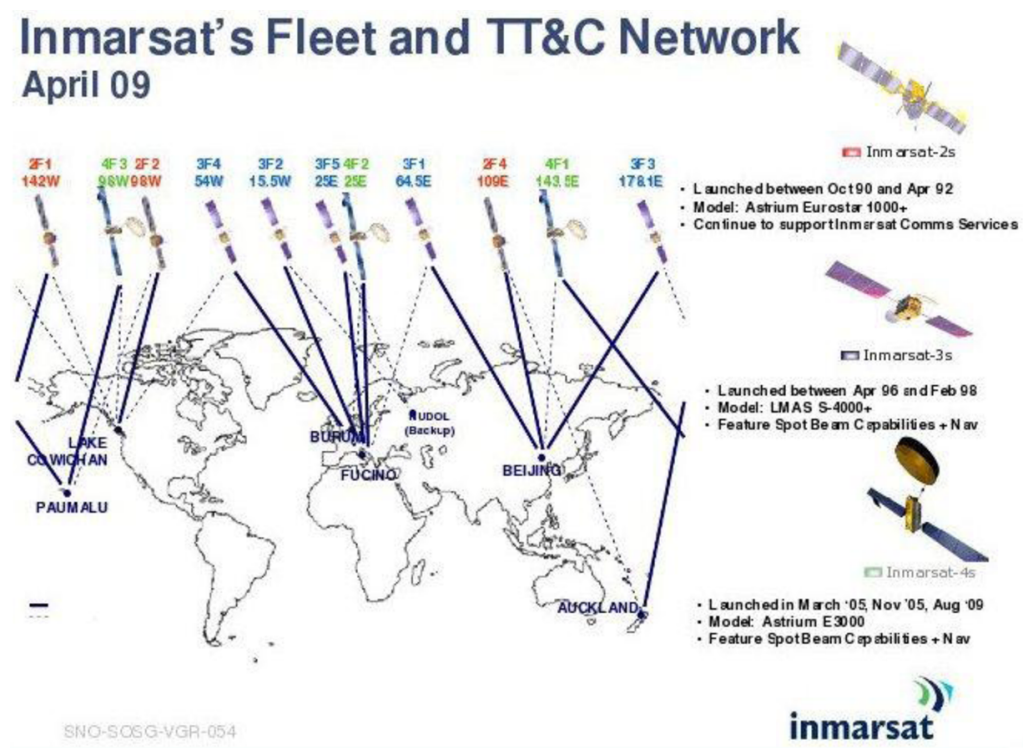




\section{Country Codes}

The permanent telephone country code for calling Inmarsat destinations is 870 SNAC (Single Network Access Code). The 870 number is an automatic locator; you don't have to know to which satellite the destination Inmarsat terminal is logged in.

\section{Gateways}

The fixed part of the ground segment comprises of one or more gateways to access the space segment, transport the necessary signalling, control and communications and support inter/intra system mobility. The location of gateway depends on operator's preferred selection criteria. Factors considered include proximity to the terrestrial traffic, desired network connectivity and routing arrangements.

Gateways comprise of a radio system to support transport over the satellite system and a network switching system to interconnect to the terrestrial network. The satellite mobile phone uses the IP protocol.

The gateways are interfaced to various terrestrial networks such as Public Switched Telephone Network (PSTN), Public Land Mobile Network (PLMN) such as GPRS or UMTS, Internet, private network, etc. The service providers, responsible for end-to-end service provision, access the satellite network through the appropriate interfaces.

\section{BGAN (Global Voice and Broadband Data)}

BGAN use the Inmarsat-4 satellites and are used from laptop-sized terminals.

I-4 traffic is transported mainly in the form of IP (Internet Protocol) data packet, thereby increasing the capacity of the Inmarsat network to provide advanced digital mobile communications: Email, Internet access, Secure VPN, Telephony, VoIP, SMS, Videoconferencing, Live video streaming, File transfer, Time critical data transfer, Remote surveillance. The Inmarsat IsatPhone represents a cell phone option at low cost. The service is initially available in Asia, Africa and the Middle East.

FleetBroadband is the tradename of the BGAN technology when it is implemented in the maritime field.

SwiftBroadband is the tradename of the BGAN technology when it is implemented in the aeronautical field (Onair and Aeromobile are using Inmarsat for aircraft communications) Inmarsat Ventures Plc (Inmarsat) did not report having the roaming agreements with other operators except of SMS interconnection. 


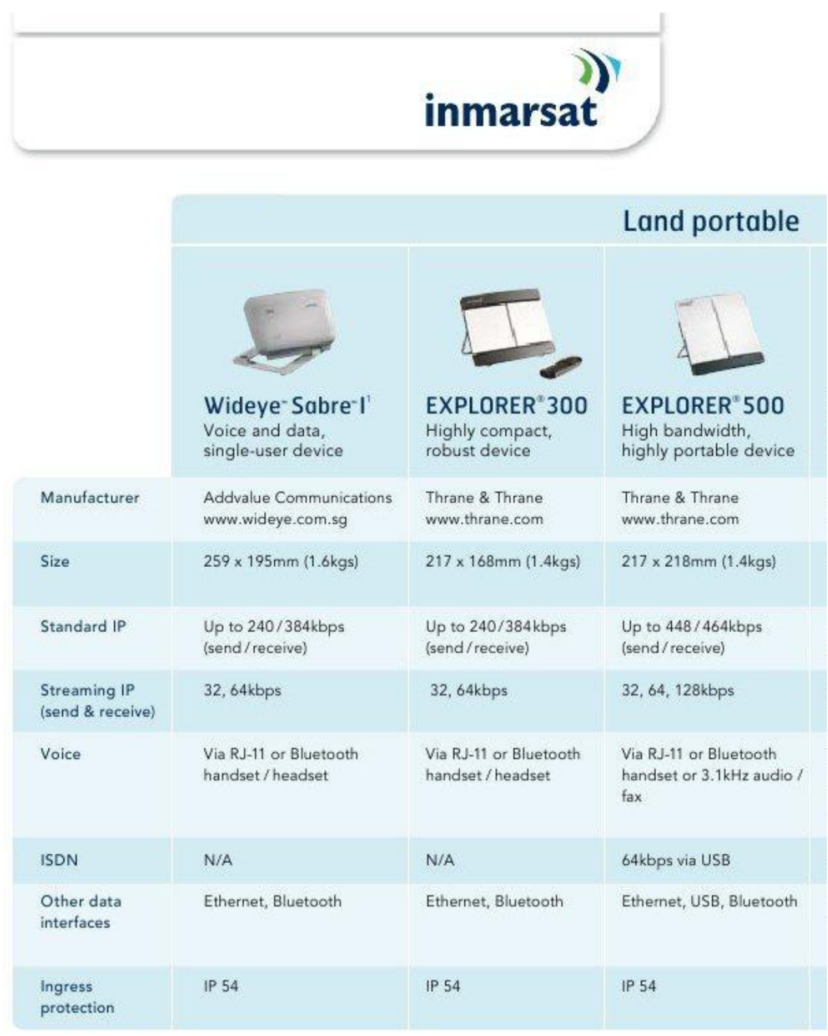

Inmarsat and KPN have agreed that all SCCP traffic towards Inmarsat is routed via KPN. For this purpose the INMARSAT global title range for SCCP routing is linked to the KPN network. The KPN and Inmarsat networks are interconnected for the distribution of SCCP traffic.

This document will describe the number ranges involved, the translation rules, the platform releases and contact persons. Note that this document only relates to SCCP traffic for SMS, since Inmarsat has not implemented any roaming due to both terminal and air-interface incompatibility with other terrestrial based GSM/3G operators.

It is not required for a network operator to have an AA.19 interworking agreement with either KPN or Inmarsat for sending SMS traffic towards Inmarsat subscribers. 


\section{Ground Implementation of the Number Continuity}

\subsection{Case of Standard GSM Core Network}

In [4, chapter 2], one finds the very detailed message flow for a classical single or multi-imsi virtual roaming service implementation. Figure 5 is the signaling flow for Globalstar's GSM based handsets, which use a GSM core network. It would be the same thing for Iridium, Inmarsat or Thuraya which also have a GSM core network. The Roaming Hub has a table to correspond the "IMSI auxiliary" (received from the satphone) to the "IMSI nominal" (the GSM). In (1), the VLR sends a SEND AUTHENTICATION to request an authentication "challenge". To secure the system, this is looped back (2) to the Globalstar HLR, which returns a Challenge (RAND and SRES) back to the satphone (3) and (4) through the Roaming Hub. If the IMSI auxiliary in the satphone compute and match the challenge, the signalling will continue with an UPDATE LOCATION req (5) which is transformed by the Roaming Hub (IMSI auxiliary replaced by IMSI nominal). The GSM HLR will transfer in an INSERT SUBSCRIBER DATA the MSISDN (GSM) (7) which is assigned (8) to the satphone.

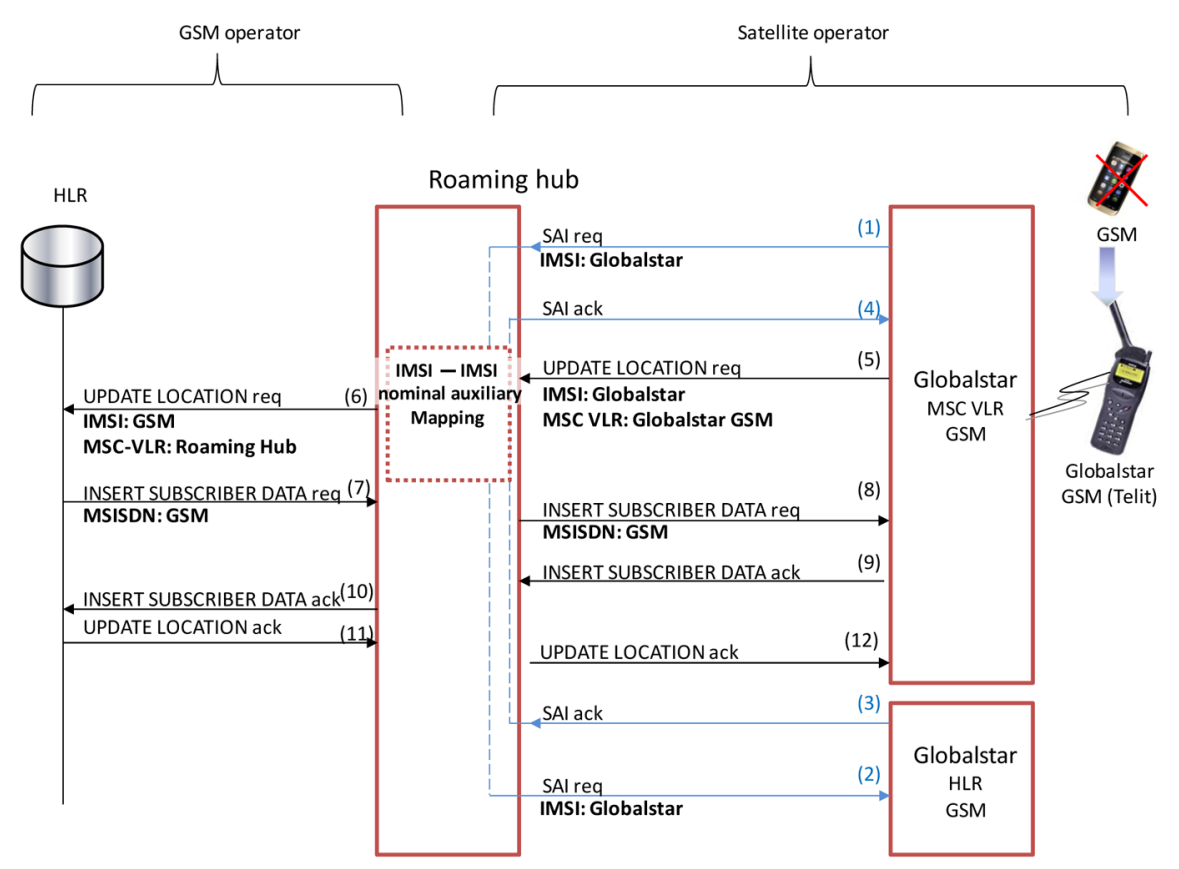

Figure 5 Standard GSM core network. 


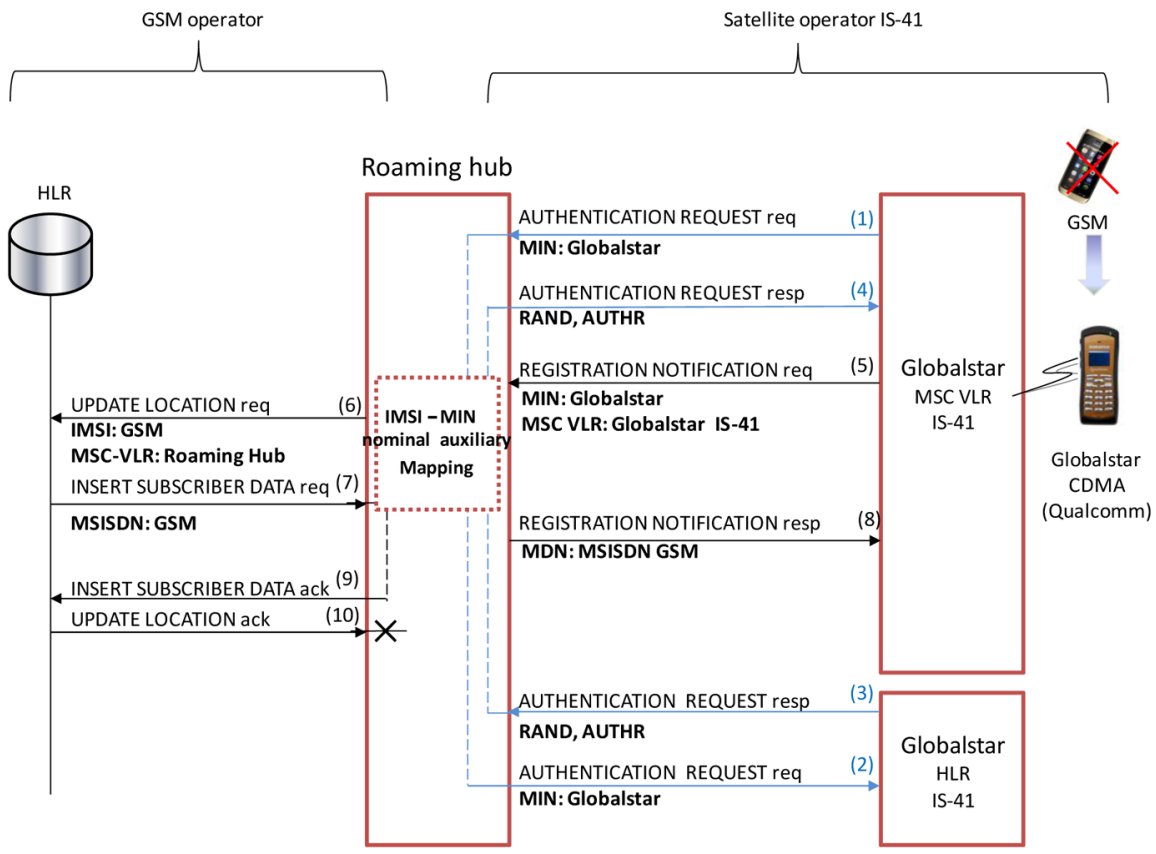

Figure 6 IS-41 core network.

\subsection{Case of IS-41 Core Network}

It is the same signaling flow as for GSM core networks, except that the Roaming Hub must translate certain IS-41 messages to their GSM counterpart. Figure 6 follows the same pattern and gives a quick equivalence (MIN is the IS-41 equivalent of IMSI). Here we only give the principle of the authentication and registration step, in future work we will show in particular the detailed message flows for voice calls and SMS.

\section{References}

[1] P. Nicopolitidis, M. S. Obaidat, G. I. Papadimitriou, and A. S. Pomportsis. Wireless Networks, Chap. 7, Wiley, 2003.

[2] X. Lagrange, P. Godlewski, S. Tabbane. Réseaux GSM-DCS, Chapters 5 and 10 ("Handover"), Hermès, 1997.

[3] Wolfram Mathematica website, integrals.wolfram.com (Note: this gives a real value function only for $|e|>1$ ).

[4] A. Henry-Labordère. Virtual Roaming Systems for GSM, GPRS and UMTS, Wiley, 2009. 
[5] Maurice Roy. Mcanique, Vol. I Corps Rigides, Vol. II Milieux continus, Dunod, 1965 (in particular, Vol. I, pp. 62-65).

\section{Biography}

Arnaud Henry-Labordère is a graduate engineer from Ecole Centrale de Paris (1966), Ph.D. (Mathematics, USA, 1968). He was professor of Operations Research at Ecole Nationale des Ponts et Chaussées during 25 years, as well as at Ecole Nationale des Mines de Paris. He is currently Visiting Professor at Prism-CNRS. He started at IBM research (1967) and founded three companies: FERMA (voice mail systems in 1983), Nilcom (first SMS network in 1999) and currently Halys (telecom equipments). He is the author of eight books (six in maths, two in telecoms) and has been granted 85 patents. 